\title{
Management of pregnancies with high risk of severe haemolytic disease of the fetus
}

\author{
Tiran Dias ${ }^{1}$ \\ Sri Lanka Journal of Obstetrics and Gynaecology 2012; 34: 176-178
}

\section{Introduction}

Fetal anaemia can be secondary to both immune and non-immune conditions. Maternal sensitization happens when a pregnant woman is carrying a fetus with paternally derived red-cell antigen that is foreign to the mother. Since primary immune response takes time to activate, first pregnancy usually does not affect. In subsequent pregnancies antibody response is rapid and antibodies are formed in greater concentration. These antibodies may cross the placenta, bind to antigens present on the fetal erythrocytes, and cause hemolysis, hydrops fetalis, and fetal death.

Rhesus (Rh) alloimmunization is the most common cause for severe haemolytic disease of the fetus. Incidence of fetal anaemia due to Rh incompatibility has been declining over the past decades with the introduction of anti-D prophylaxis in 1969. Despite routine use of anti-D prophylaxis, it has been estimated that nearly 600-700 new cases of rhesus sensitization still occur each year in the $\mathrm{UK}^{1}$. This is primarily due to silent fetomaternal heamorrhage and inadequate dose of anti-D prophylaxis.

Haemolytic disease of the fetus due to other redcell antibodies such as anti-c, K1 (Kell), Duffy and antiE have gained importance as Rhesus D alloimmunization has become less of a problem. Non-immune conditions such as massive fetomaternal hemorrhage and fetal infection following human parvovirus B19 (HPVB19) are other important causes of fetal anaemia.

\section{Prediction of fetal anaemia}

Primary objective of monitoring at risk fetus of severe haemolytic disease is to timely diagnosis of anaemia which needs fetal blood transfusion and delivers them at appropriate time. Rh sensitized mothers need regular Rh antibody level assessment and antibody level $>4$ IU ( 1:32) is considered significant. Antibody level $>10$ IU $(\sim 1: 64)$ puts fetus at risk of haemolytic disease and $>15$ IU $(\sim 1: 132)$ confers very high risk of fetal anaemia. Fetus is at greater risk of anaemia if the antibody levels are $>10$ IU ( 1:64) at the beginning of the pregnancy or rise suddenly. Rising antibody titre warrants urgent referral to a fetal medicine unit for further management.

Invasive methods such as amniocentesis and fetal blood sampling (FBS) were the choices in past to screen the high risk fetuses for severe haemolytic disease. Amniocentesis indirectly identifies level of fetal anaemia by quantifying bilirubin (delta optical density 450 , OD450) present in the amniotic fluid as a result of fetal hemolysis. It does not provide information about the hemopoietic response of the fetus and serial procedures are usually required to monitor the pregnancy. This method shows a lack of accuracy before 27 weeks of gestation and in predicting the severity of anaemia ${ }^{2,3}$. Moreover, in pregnancies affected by Kell alloimmunization or HPVB19 infection, the correlation between the delta optical density 450 (OD450) and anaemia is poor as the anaemia is caused by decreased hematopoiesis rather than red blood cell destruction $^{4,5}$. Cordocentesis and FBS can identify fetal anaemia reliably but this procedure is known to associate with complications such as, bleeding from the cord puncture site, transient bradycardia, worsening of fetal alloimmunization and fetal demise ${ }^{6}$.

It has been clearly reveled that cerebral arteries respond quickly to hypoxemia due to the strong dependence of brain tissue on oxygen. Peak systolic velocity of the middle cerebral artery (MCA-PSV), as measured by Doppler ultrasonography, is increased in fetuses with anaemia ${ }^{7}$. The advantage of studying the MCA is easy visualization with an angle of close to 0 degrees between the ultrasound beam and the direction of blood flow and this measurement has low intra-observer and inter-observer variability ${ }^{7}$. The measurement of the MCA-PSV can predict the presence of moderate or severe anaemia in fetuses with a sensitivity of $100 \%$ and a false positive rate of $12 \%$ of a PSV threshold of $1.5 \mathrm{MoM}^{8}$ (Figure 1).

\footnotetext{
${ }^{1}$ Consultant Obstetrician and Gynaecologist District General Hospital, Ampara Sri Lanka.
} 


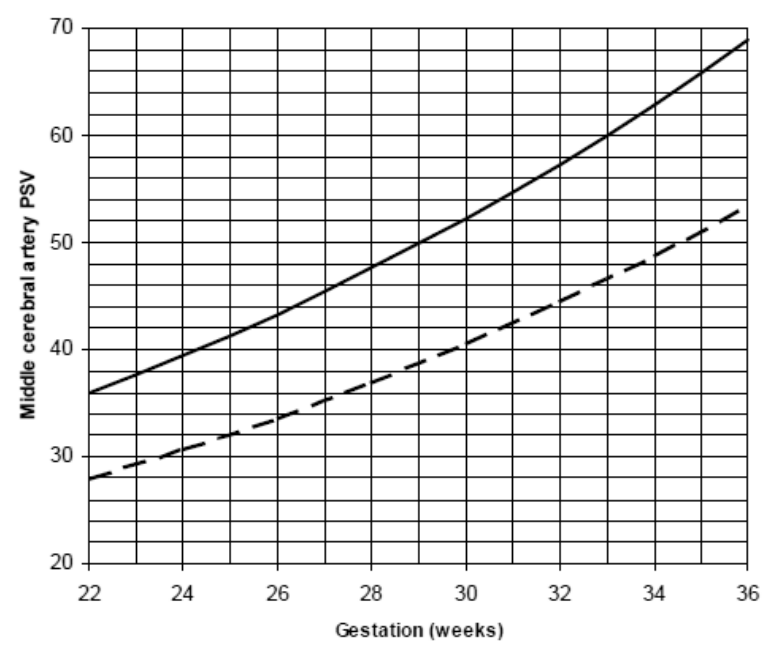

Figure 1. MCV-PSV reference shows median for gestation (interrupted line) and 1.5 MoM action level (solid line). This chart is only applicable for angle corrected velocity data. (Adopted from Mari G et al. N Engl J Med 2000; 342: 9-14).

\section{Technique}

An axial section of the brain, including the thalami and the cavum septi pellucidi, should be obtained. Then the circle of Willis should be visualized and the middle cerebral artery of one side needs to be examined close to its origin in the internal carotid artery as the systolic velocity decreases with distance from the point of origin of this vessel. The angle between the ultrasound beam and the direction of blood flow should be kept as close as possible to 0 degrees. The highest point of the wave form (peak systolic velocity) needs to be measured (Figure 2).

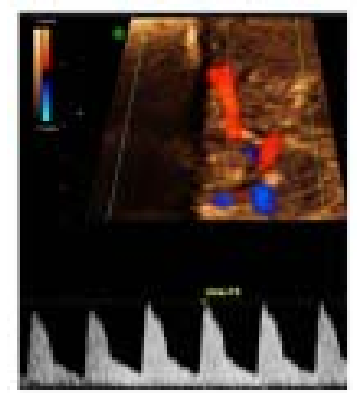

- Transverse section of the head

- Locahe sphenoid bones (wingl

- Colour bo locate Cirde of Whasis

- Zoom calour box en MCA

- Sample provimal end of MCA.

- Gate in centre of versel

- Angie contection

- Measure PSV daring feral rest

Figure 2. Measurement of peak systolic velocity of the middle cerebral artery (MCA-PSV).

\section{Intrauterine transfusion (IUT)}

In the absence of systematic referral system and prompt IUT facilities, severe Rh alloimmunization confers high perinatal morbidity and mortality. IUT was not routinely performed in Sri Lanka and it has been recently started ${ }^{9}$. The first intrauterine fetal blood transfusion was performed in 1963 by Liley, utilizing an intraperitoneal approach ${ }^{10}$.With time the procedure has been modified to involve an intravascular approach the safety of which has increased with improvements in ultrasound technology ${ }^{11}$. Recognized intravascular techniques utilize for intrauterine blood transfusions include cordocentesis (usually into the umbilical vein either at the point where the cord inserts into the placenta or into the baby's liver, or into a free loop of cord) or into a blood vessel in the baby's liver (intrahepatic transfusion) (Figure 3).

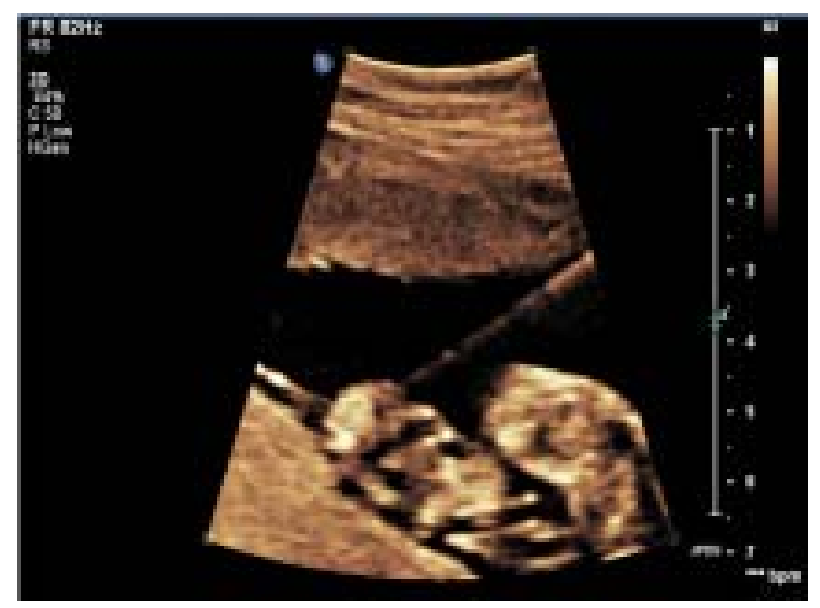

Figure 3. Cordocentesis and fetal blood transfusion into the placental cord insertion.

Intrauterine blood transfusion is associated with complications such as preterm prelabour rupture of membranes, chorioamnionitis, preterm labour, and fetal distress. These complication may necessitate emergency delivery and result in preterm birth and its' complications. Often a baby will require repeated transfusions during the course of a pregnancy and these repeated procedures will promote additional antibody formation. Increasing antibody sensitization further exacerbates the fetal haemolytic process. Moreover, Inutero transfusion of blood suppresses the baby's erythropoiesis and this suppression requires repeat transfusions during the course of the pregnancy, and the need for top-up transfusions after the baby's birth.

\section{References}

1. Rodeck $\mathrm{CH}$, Deans A. Red cell alloimmunisation. In Fetal Medicine: Basic Science and Clinical Practice, Rodeck $\mathrm{CH}$, Whittle MJ (eds). Churchill Livingstone: Edinburgh, UK, 1999.

2. Rahman F, Detti L, Ozcan T, Khan R, Manohar S, Mari G. 
Can a single measurement of amniotic fluid delta optical density be safely used in the clinical management of Rhesusalloimmunised pregnancies before 27 weeks' gestation? Acta Obstet Gynecol Scand 1988; 77: 804-7.

3. Nicolaides $\mathrm{KH}$, Rodeck $\mathrm{CH}$, Mibashan RS, Kemp JR. Have Liley charts outlived their usefulness? Am J Obstet Gynecol 1986; 155: 90-4

4. Freire-Lizama T, Oepkes D. Management of Kell alloimmunized pregnancies. Fetal Maternal Med Rev 2002; 13: 87-102.

5. Brown K, Young N. Parvovirus B19 infection and hematopoiesis. Blood Rev 1995; 9: 176-82.

6. Ludomirsky A, Weiner S, Aslmead GG, Librizzi RJ, Bolognese RJ. Percutaneous fetal umbilical blood sampling. Procedure safety and normal fetal hematologic indices. Am J Perinatol 1988; 5: 264-6.

7. Mari G, Andrignolo A, Abuhamad AZ, et al. Diagnosis of fetal anaemia with Doppler ultrasound in the pregnancy complicated by maternal blood group immunization. Ultrasound Obstet Gynecol 1995; 5: 400-5.

8. Mari G, Deter RL, Carpenter RL, Rahman F, Zimmerman R, Moise KJ Jr, Dorman KF, Ludomirsky A, Gonzalez R, Gomez R, Oz U, Detti L, Copel JA, Bahado-Singh R, Berry $S$, Martinez-Poyer J, Blackwell SC. Noninvasive diagnosis by Doppler Ultrasonography of fetal anaemia due to maternal red-cell alloimmunisation. Collaborative Group for Doppler Assessment of the Blood Velocity in Anemic Fetuses. N Engl J Med 2000; 342: 9-14.

9. Dias T, Senanayake H, Ruwanpura L, Pathirana P, Dissanayake P. Intra-uterine fetal blood transfusion. Ceylon Med J. 2012; 57(2): 93-4.

10. Liley AW. Intrauterine transfusion of foetus in hemolytic disease. BMJ 1963; 2: 1107-9.

11. Rodeck CH, Kemp JR, Holman CA, Whitmore DN, Karnicki J, Austin MA. Direct intravascular fetal blood transfusion by fetoscopy in severe rhesus isoimmunisation. Lancet 1981; 1: 625-7. 